Psychological Medicine

cambridge.org/psm

\section{Original Article}

${ }^{\star}$ Contributed equally.

Cite this article: Pillinger T, McCutcheon RA, Howes OD (2022). Variability of glucose, insulin, and lipid disturbances in first-episode psychosis: a meta-analysis. Psychological Medicine 1-7. https://doi.org/10.1017/ S0033291721005213

Received: 19 September 2021

Revised: 19 November 2021

Accepted: 30 November 2021

\section{Key words:}

cholesterol; diabetes; metabolic; psychosis; schizophrenia; variability

\section{Author for correspondence:}

Toby Pillinger,

E-mail: toby.pillinger@kcl.ac.uk

\title{
Variability of glucose, insulin, and lipid disturbances in first-episode psychosis: a meta-analysis
}

Toby Pillinger ${ }^{1,2,3}$ (D), Robert A. McCutcheon ${ }^{1,2,3, *}$ and Oliver D. Howes ${ }^{2,3,4,5, *}$

${ }^{1}$ Department of Psychosis Studies, Institute of Psychiatry, Psychology and Neuroscience, King's College of London, London, UK; ${ }^{2}$ Psychiatric Imaging Group, MRC London Institute of Medical Sciences, Hammersmith Hospital, Imperial College London, London, UK; ${ }^{3}$ Faculty of Medicine, Institute of Clinical Sciences, Imperial College London, London, UK; ${ }^{4}$ Department of Psychological Medicine, Institute of Psychiatry, Psychology and Neuroscience, King's College of London, London, UK and ${ }^{5} \mathrm{H}$ Lundbeck A/s, 3 Abbey View, Everard Close, St Albans AL1 2PS, UK

\begin{abstract}
Background. First-episode psychosis (FEP) is associated with metabolic alterations. However, it is not known if there is heterogeneity in these alterations beyond what might be expected due to normal individual differences, indicative of subgroups of patients at greater vulnerability to metabolic dysregulation.

Methods. We employed meta-analysis of variance, indexed using the coefficient of variation ratio (CVR), to compare variability of the following metabolic parameters in antipsychotic naïve FEP and controls: fasting glucose, glucose post-oral glucose tolerance test (OGTT), fasting insulin, insulin resistance, haemoglobin $\mathrm{A}_{1 \mathrm{c}}\left(\mathrm{HbA}_{1 \mathrm{c}}\right)$, total-cholesterol, low-density lipoprotein (LDL)-cholesterol, high-density lipoprotein (HDL)-cholesterol, and triglycerides. Standardised mean difference in metabolic parameters between groups was also calculated; meta-regression analyses examined physiological/demographic/psychopathological moderators of metabolic change.

Results. Twenty-eight studies were analysed (1716 patients, 1893 controls). Variability of fasting glucose $[\mathrm{CVR}=1.32 ; 95 \%$ confidence interval (CI) $1.12-1.55 ; p=0.001]$, glucose postOGTT $(C V R=1.43 ; 95 \%$ CI $1.10-1.87 ; p=0.008)$, fasting insulin $(\mathrm{CVR}=1.31 ; 95 \% \mathrm{CI}$ $1.09-1.58 ; p=0.01)$, insulin resistance $(\mathrm{CVR}=1.34 ; 95 \% \mathrm{CI} 1.12-1.60 ; p=0.001), \mathrm{HbA}_{1 \mathrm{c}}$ $(\mathrm{CVR}=1.18 ; 95 \%$ CI $1.06-1.27 ; p<0.0001)$, total-cholesterol $(\mathrm{CVR}=1.15 ; 95 \%$ CI $1.01-$ $1.31 ; p=0.03)$, LDL-cholesterol (CVR $=1.28$; 95\% CI 1.09-1.50; $p=0.002)$, and HDL-cholesterol $(\mathrm{CVR}=1.15 ; 95 \%$ CI $1.00-1.31 ; p<0.05)$, but not triglycerides, was greater in patients than controls. Mean glucose, glucose post-OGTT, fasting insulin, insulin resistance, and triglycerides were greater in patients; mean total-cholesterol and HDL-cholesterol were reduced in patients. Increased symptom severity and female sex were associated with worse metabolic outcomes.

Conclusions. Patients with FEP present with greater variability in metabolic parameters relative to controls, consistent with a subgroup of patients with more severe metabolic changes compared to others. Understanding determinants of metabolic variability could help identify patients at-risk of developing metabolic syndrome. Female sex and severe psychopathology are associated with poorer metabolic outcomes, with implications for metabolic monitoring in clinical practice.
\end{abstract}

(c) The Author(s), 2022. Published by Cambridge University Press. This is an Open Access article, distributed under the terms of the Creative Commons Attribution licence (http://creativecommons.org/licenses/by/4.0/), which permits unrestricted re-use, distribution and reproduction, provided the original article is properly cited.

\section{CAMBRIDGE UNIVERSITY PRESS}

\section{Introduction}

People with psychotic disorders die 15 years earlier than members of the general population (Crump, Winkleby, Sundquist, \& Sundquist, 2013). Most of this excess mortality is secondary to physical health conditions, predominantly cardiometabolic disease, highlighting the importance of identifying underlying factors (Osby, Correia, Brandt, Ekbom, \& Sparen, 2000; Pillinger et al., 2019b; Pillinger et al., 2019c; Pillinger, D’Ambrosio, McCutcheon, \& Howes, 2019a). Although lifestyle and medication play a key role in the metabolic disturbances seen in individuals with psychosis (Pillinger et al., 2020), meta-analyses have observed glucose and lipid alterations from psychosis onset and in the absence of antipsychotic treatment (Greenhalgh et al., 2017; Perry, McIntosh, Weich, Singh, \& Rees, 2016; Pillinger et al., 2017a, 2019a; Pillinger, Beck, Stubbs, \& Howes, 2017b). Moreover, these alterations persist when patients and controls are matched for factors associated with metabolic function e.g. diet, physical activity, and body mass index (BMI) (Pillinger et al., 2017a, 2017b).

Thus, there is evidence that psychosis is associated with metabolic alterations independent of common risk factors. However, it is not known if psychosis-associated metabolic alterations manifest as a uniform effect (i.e. mean metabolic levels are increased in patients with similar variability of data around the mean for both patients and controls) or, alternatively, if there is 
heterogeneity in this effect amongst patients beyond what might be expected due to normal individual differences (i.e. mean metabolic levels are increased in patients but also with increased variability of data in patients compared with controls). We set out to address this question by performing a meta-analysis of variability, as previously employed to examine peripheral immune marker and brain structural variability in first-episode psychosis (FEP) (Brugger \& Howes, 2017; Pillinger et al., 2019b). If there is heterogeneity in metabolic alterations amongst patients, e.g. because they are seen only in a subgroup of patients, then there will be greater metabolic variability in patients relative to controls. Conversely, if metabolic alterations are a homogenous component of the pathophysiology of psychosis, reduced or similar metabolic variability in patients compared with controls would be predicted, reflecting homogeneity in pathophysiology.

Since publication of previous meta-analyses assessing mean differences in metabolic parameters between FEP and controls (Pillinger et al., 2017a, 2017b), up to eight new case-control studies have been published. Therefore, we also aimed to perform updated meta-analyses of mean differences in metabolic parameters between FEP and controls. The factors underlying metabolic alterations are not clear, and between-study inconsistency has been highlighted as a limitation of previous meta-analyses (Reynolds, 2021), so we additionally investigated factors that might underlie effects and explain inconsistency.

\section{Methods}

A systematic review was performed according to Preferred Reporting Items for Systematic Reviews and Meta-Analyses and Meta-Analysis of Observational Studies in Epidemiology guidelines (Moher, Liberati, Tetzlaff, Altman, \& Group, 2009; Stroup et al., 2000) (online Supplementary eAppendix 1), following an a priori protocol (PROSPERO: CRD 42021271674). The PubMed, EMBASE, and PsycINFO were searched from inception to August (week 1) 2021 for appropriate articles (online Supplementary eAppendix 2).

\section{Selection criteria}

Inclusion criteria were: (1) patients with diagnoses of schizophrenia, schizoaffective disorder, schizophreniform disorder, schizophrenia spectrum and psychotic disorder not otherwise specified as defined by the Diagnostic and Statistical Manual of Mental Disorders (DSM) or International Statistical Classification of Diseases (ICD); (2) first episode of illness defined as patients within 5 -years of first presentation; (3) participants aged above 18 years; (4) antipsychotic-naïve/ $\leqslant 2$ weeks of antipsychotic treatment; (5) a healthy control group; (6) studies assessing plasma fasting glucose, the oral glucose tolerance test (OGTT), fasting insulin, insulin resistance (measured using homoeostatic model assessment for insulin resistance, HOMA-IR), haemoglobin $\mathrm{A}_{1 \mathrm{c}}\left(\mathrm{HbA}_{1 \mathrm{c}}\right)$, totalcholesterol, low-density lipoprotein (LDL)-cholesterol, high-density lipoprotein (HDL)-cholesterol, and triglyceride levels. Exclusion criteria were: (1) substance/medication-induced psychosis and (2) absent mean and/or variance data.

\section{Recorded variables}

Data were extracted as follows: author, publication year, mean (with standard deviation) measure of metabolic parameter, patient/control number, diagnosis, antipsychotic naïve-status, proportion of males/smokers/non-Caucasians, illness duration, and symptom rating (total Positive and Negative Syndrome Scale (PANSS) scores) (Kay, Fiszbein, \& Opler, 1987).

\section{Statistical analysis}

For difference in variability, the natural log of the ratio of estimates of the population standard deviations scaled to mean for each group was calculated, giving the natural logarithm of the ratio of estimates of population coefficients of variation (lnCVR), as previously described (online Supplementary eAppendix 3) (Pillinger et al., 2019b). To aid interpretation, lnCVR effect sizes were transformed to a linear scale; thus, coefficient of variation ratio $(\mathrm{CVR})>1$ indicates greater relative variability in patient groups, and $\mathrm{CVR}<1$ indicates lower relative variability in patient groups.

Standardised mean differences (SMD) in metabolic measurements between patients and controls were also calculated. In expectation of inconsistency across studies, a random-effects model was used; inconsistency was assessed using Cochran $Q$ and $I^{2}$ statistics (Bowden, Tierney, Copas, \& Burdett, 2011; Higgins, Thompson, Deeks, \& Altman, 2003). If more than 10 studies were analysed, publication bias was assessed using the Egger test of the intercept (Egger et al., 1997) and represented diagrammatically with funnel plots.

To determine if findings were influenced by confounding, we performed three sensitivity analyses: (1) excluding studies where patients received antipsychotic treatment; (2) excluding studies which included patients other than those with schizophrenia; and (3) excluding studies identified as outliers following leave-one-out diagnostics (Viechtbauer \& Cheung, 2010).

Meta-regression analyses were performed to explore the effects of patient age, proportion of males, BMI, proportion of people of non-Caucasian ethnicity, proportion of smokers, illness duration, and total PANSS score on mean metabolic effect size magnitudes. A two-tailed $p<0.05$ was deemed significant for all analyses.

\section{Results}

\section{Retrieved studies}

The search identified 7975 citations. After exclusion of studies not meeting criteria or reporting overlapping data, 28 studies met inclusion criteria and were analysed. The search process is demonstrated in online Supplementary eAppendix 2, and final studies selected are summarised in online Supplementary eTable 1. The overall sample included 1716 patients and 1893 controls.

\section{Fasting glucose concentration}

Fasting glucose was analysed using data from 22 studies comprising 1403 patients and 1407 controls. Fasting glucose was more variable in patients compared with controls $[\mathrm{CVR}=1.32 ; 95 \%$ confidence interval (CI) 1.12-1.55; $p=0.001$ ] (Fig. 1, online Supplementary eTable 2, eAppendix 4). Mean fasting glucose was also elevated in patients $(\mathrm{SMD}=0.18 ; 95 \%$ CI $0.05-0.31$; $p=0.007$ ) (Fig. 2, online Supplementary eTable 2, eAppendix 4). Between-study inconsistency was not significant $\left(I^{2}\right.$ value $=$ 18.56\%; Cochrane $Q=26.91, p=0.14$ ). Funnel plot inspection (online Supplementary eAppendix 4) suggested publication bias was not significant (Egger $p=0.86$ ). Restricting analyses to 


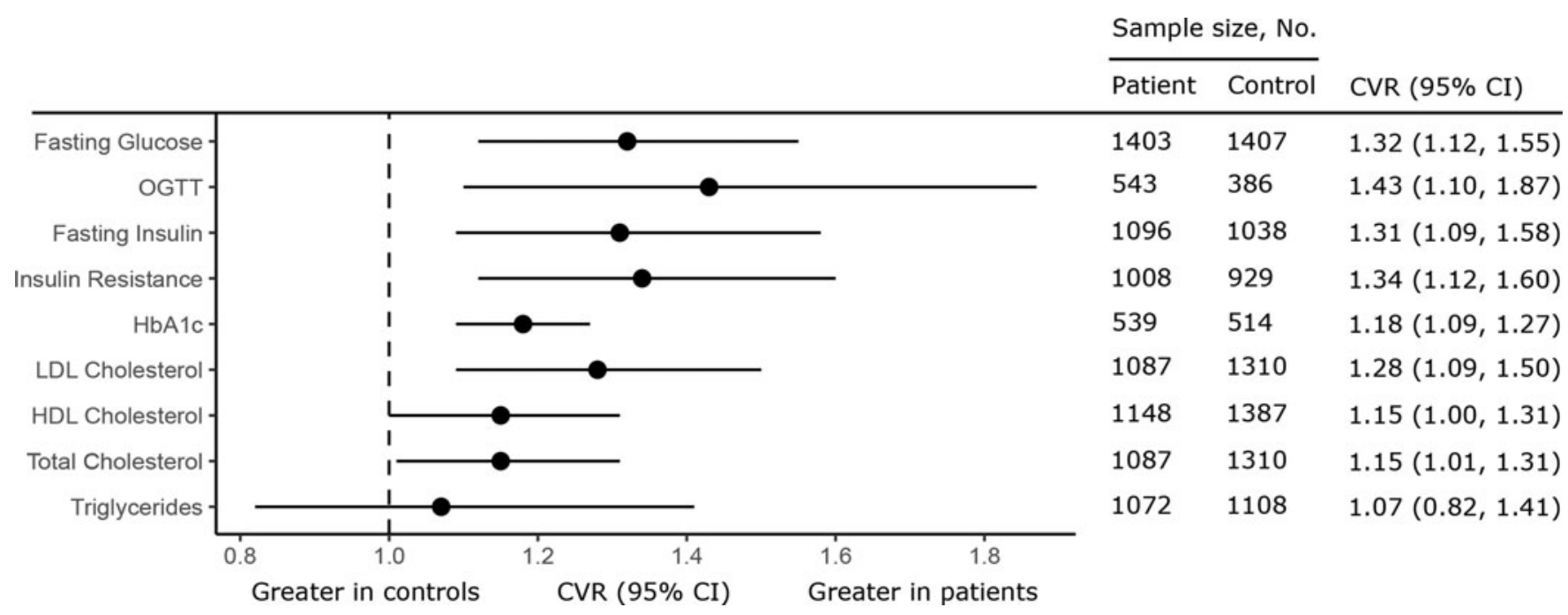

Fig. 1. Forest plot showing summary effect sizes for mean-scaled variability of glucose, insulin, and lipid parameters in antipsychotic naïve FEP compared with healthy controls. Each circle shows the summary effect size (CVR); the horizontal line running through each square illustrates the width of the overall $95 \%$ CI. The CVR was significantly increased for fasting glucose, glucose after the OGTT, fasting insulin, insulin resistance as measured using HOMA-IR, HbA ${ }_{1 \mathrm{C}}$, LDL-cholesterol, HDL-cholesterol, and total-cholesterol, indicating greater variability in these metabolic parameters in patients compared with controls. There was no significant difference in CVR for triglyceride levels in patients compared with controls.

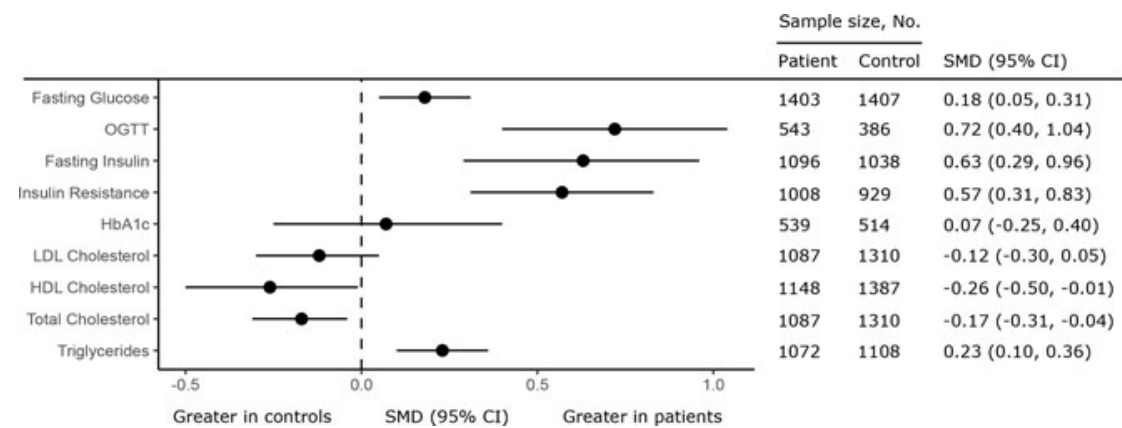

Fig. 2. Forest plot showing summary effect sizes for mean differences in glucose, insulin, and lipid parameters in antipsychotic naïve FEP compared with healthy controls. Each circle shows the summary effect size (SMD); the horizontal line running through each square illustrates the width of the overall $95 \% \mathrm{Cl}$. There was a significant elevation in fasting glucose, glucose levels measured using an OGTT, fasting insulin, insulin resistance as measured using HOMA-IR, and triglyceride levels in patients compared with controls. There was a significant decrease in total and HDL-cholesterol levels in patients compared with controls. There was no significant difference in $\mathrm{HbA}_{1 \mathrm{c}}$ or LDL-cholesterol levels in patients compared with controls. antipsychotic naïve patients and those with schizophrenia did not change CVR or SMD outcomes (online Supplementary eTables 3 and 4). Exclusion of two outlying studies (online Supplementary eAppendix 4) for the SMD analysis did not alter results (online Supplementary eTable 5); no CVR outliers were identified. Meta-regression of effect size for mean fasting glucose on patient age, sex, BMI, smoking-status, and illness duration did not yield significant results (online Supplementary eTable 6). However, increased symptom severity was associated with larger fasting glucose effect size $(z=2.10, r=0.97, p=0.04)$ (online Supplementary eAppendix 4). There were insufficient studies to explore the influence of ethnicity on glucose outcomes.

\section{Glucose concentration after OGTT}

Plasma glucose post-OGTT was analysed using data from six studies comprising 543 patients and 386 controls. Glucose concentration post-OGTT was more variable in patients compared with controls $(\mathrm{CVR}=1.43$; 95\% CI $1.10-1.87 ; p=0.008)$ (Fig. 1, online Supplementary eTable 2, eAppendix 5). Mean glucose levels post-OGTT were also elevated in patients $(\mathrm{SMD}=0.72$; 95\% CI $0.40-1.04 ; p<0.0001$ ) (Fig. 2, online Supplementary eTable 2, eAppendix 5). There was between-sample inconsistency $\left(I^{2}\right.$ value $=79.21 \%$; Cochrane $\left.Q=22.31, p<0.01\right)$. Restricting analyses to antipsychotic naïve patients and those with schizophrenia did not change CVR or SMD outcomes (online Supplementary eTables 3 and 4). Exclusion of a single outlying study (online Supplementary eAppendix 5) for CVR and SMD analyses did not alter results (online Supplementary eTable 5). There were insufficient studies to perform meta-regression analyses.

\section{Fasting insulin concentration}

Fasting insulin concentration was analysed using data from 16 studies comprising 1096 patients and 1038 controls. Fasting insulin was more variable in patients compared with controls $(\mathrm{CVR}=$ 1.31; 95\% CI 1.09-1.58; $p=0.01$ ) (Fig. 1, online Supplementary eTable 2, eAppendix 6). Mean fasting insulin was elevated in patients compared with controls $(\mathrm{SMD}=0.63$; 95\% CI 0.29-0.96; $p=0.003$ ) (Fig. 2, online Supplementary eTable 2, eAppendix 6). There was between-sample inconsistency $\left(I^{2}\right.$ value $=91.79 \%$; Cochrane $Q=122.77, p<0.01$ ). Funnel plot inspection (online Supplementary eAppendix 6) suggested publication bias was significant (Egger $p=0.04$ ). Restricting analyses to antipsychotic naive patients and those with schizophrenia did not change CVR or SMD outcomes (online Supplementary eTables 3 and 4). Exclusion of a single outlying study (online Supplementary 
eAppendix 6) for CVR and SMD analyses did not alter results (online Supplementary eTable 5). Meta-regression of effect size for mean fasting insulin on patient age, sex, and BMI did not yield significant results (online Supplementary eTable 6). There were insufficient studies to explore the influence of other moderators.

\section{Insulin resistance}

Insulin resistance (HOMA-IR) was analysed using data from 13 studies, comprising 1008 patients and 929 controls. HOMA-IR was more variable in patients compared with controls $(\mathrm{CVR}=$ $1.34 ; 95 \% \quad$ CI 1.12-1.60; $p=0.001$ ) (Fig. 1, online Supplementary eTable 2, eAppendix 7). Mean HOMA-IR was elevated in patients compared with controls $(\mathrm{SMD}=0.57 ; 95 \% \mathrm{CI}$ $0.31-0.83 ; p<0.0001$ ) (Fig. 2, online Supplementary eTable 2, eAppendix 7). There was significant between-sample inconsistency $\left(I^{2}\right.$ value $=84.74 \%$; Cochrane $\left.Q=67.49, p<0.01\right)$. Funnel plot inspection (online Supplementary eAppendix 7) suggested publication bias was not significant (Egger $p=0.97$ ). Restricting analyses to antipsychotic naive patients and those with schizophrenia did not change CVR or SMD outcomes (online Supplementary eTables 3 and 4). Exclusion of an outlying study (online Supplementary eAppendix 7) for the SMD analysis did not alter results (online Supplementary eTable 5); no CVR outliers were identified. Meta-regression of effect size for mean HOMA-IR on patient age and BMI did not yield significant results (online Supplementary eTable 6). However, fewer males in study populations were associated with higher HOMA-IR effect size magnitudes $(z=-2.32, r=-0.98, p=0.02)$ (Fig. 3 ). There were insufficient studies to explore the influence of other moderators.

\section{$H b A_{1 c}$ levels}

$\mathrm{HbA}_{1 \mathrm{c}}$ levels were analysed using data from five studies comprising 539 patients and 514 controls. $\mathrm{HbA}_{1 \mathrm{c}}$ levels were more variable in patients compared with controls $(\mathrm{CVR}=1.18 ; 95 \% \mathrm{CI}$ 1.06-1.27; $p<0.0001$ ) (Fig. 1, online Supplementary eTable 2, eAppendix 8). However, mean $\mathrm{HbA}_{1 \mathrm{c}}$ levels were not altered in patients compared with controls $(\mathrm{SMD}=0.07 ; 95 \% \mathrm{CI}-0.25$ to $0.40 ; p=0.66$ ) (Fig. 2, online Supplementary eTable 2, eAppendix 8). There was significant between-sample inconsistency $\quad\left(I^{2} \quad\right.$ value $=77.13 \%$; Cochrane $\left.Q=23.19, \quad p<0.01\right)$. Restricting analyses to antipsychotic naïve patients did not change results for either CVR or SMD outcomes; there were insufficient studies to perform sensitivity analyses restricted to people with schizophrenia (online Supplementary eTables 3 and 4). Exclusion of a single outlying study (online Supplementary eAppendix 8) for CVR and SMD analyses did not alter results (online Supplementary eTable 5). There were insufficient studies to perform meta-regression analyses.

\section{Total-cholesterol concentration}

Total-cholesterol concentration was analysed using data from 14 studies comprising 1087 patients and 1310 controls. Total-cholesterol was more variable in patients compared with controls $(\mathrm{CVR}=1.15 ; 95 \%$ CI 1.01-1.31; $p=0.03$ ) (Fig. 1, online Supplementary eTable 2, eAppendix 9). Mean total-cholesterol was reduced in patients compared with controls $(\mathrm{SMD}=-0.17$; $95 \%$ CI -0.31 to $-0.04 ; p=0.01$ ) (Fig. 2, online Supplementary
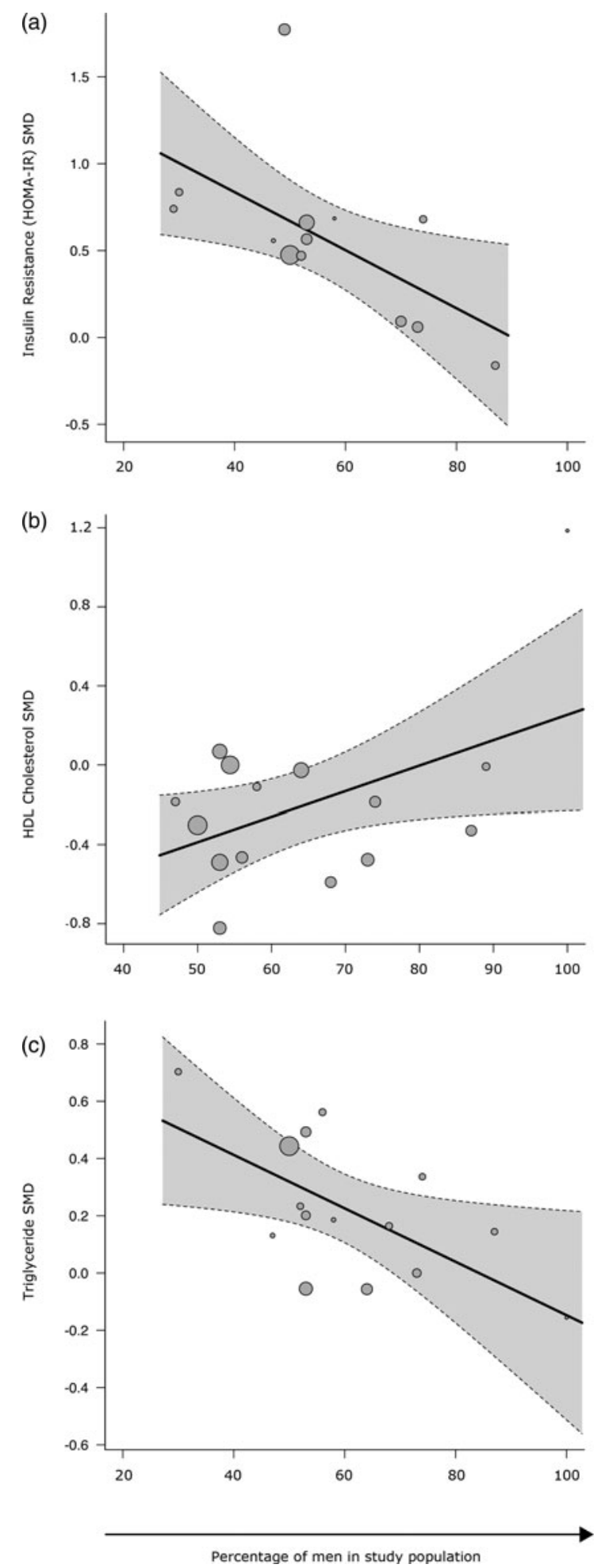

Fig. 3. Scatterplots for regression of SMD between patients and controls for metabolic parameters on percentage of men in the patient population. (a) Percentage of males in the sample was significantly associated with lower effect size for insulin resistance (as measured using HOMA-IR) effect size ( $z=-2.32, r=-0.98, p=0.02) ;(b)$ percentage of males was significantly associated with lower HDL-cholesterol effect size $(z=2.02, r=0.97, p=0.04)$; and $(c)$ percentage of males was significantly associated with lower triglyceride effect size $(z=-2.18, r=-0.98, p=0.03)$. Each circle represents a study, its size corresponding to the study weight. Single straight line represents the regression coefficient, the curved dotted lines the $95 \% \mathrm{Cl}$. 
eTable 2, eAppendix 9). There was significant between-sample inconsistency $\left(I^{2}\right.$ value $=50.55 \%$; Cochrane $\left.Q=29.17, p<0.01\right)$. Funnel plot inspection (online Supplementary eAppendix 9) suggested publication bias was not significant (Egger $p=0.73$ ). Restricting analyses to antipsychotic naïve patients did not change CVR or SMD outcomes (online Supplementary eTable 4). However, restricting analyses to those patients with schizophrenia resulted in no differences in variability or mean difference in total-cholesterol between patients and controls (online Supplementary eTable 3). Exclusion of an outlying study (online Supplementary eAppendix 9) for the CVR analysis did not alter results (online Supplementary eTable 5); no SMD outliers were identified. Meta-regression of effect size for mean total-cholesterol on patient age, sex, and BMI did not yield significant results (online Supplementary eTable 6). There were insufficient studies to explore the influence of other moderators.

\section{LDL-cholesterol concentration}

LDL-cholesterol concentration was analysed using data from 11 studies comprising 1087 patients and 1310 controls. LDL-cholesterol was more variable in patients compared with controls $(\mathrm{CVR}=1.28$; 95\% CI 1.09-1.50; $p=0.002$ ) (Fig. 1, online Supplementary eTable 2, eAppendix 10). However, mean LDL-cholesterol was not altered in patients compared with controls $(\mathrm{SMD}=-0.12$; $95 \%$ CI -0.30 to $0.05 ; p=0.17$ ) (Fig. 2, online Supplementary eTable 2, eAppendix 10). There was between-sample inconsistency $\left(I^{2}\right.$ value $=66.11 \%$; Cochrane $\left.Q=33.45, p<0.01\right)$. Funnel plot inspection (online Supplementary eAppendix 10) suggested publication bias was not significant (Egger $p=0.73$ ). Restricting analyses to antipsychotic naïve patients and those with schizophrenia did not change CVR or SMD outcomes (online Supplementary eTables 3 and 4). Exclusion of a single outlying study (online Supplementary eAppendix 10) for CVR and SMD analyses did not alter results (online Supplementary eTable 5). Meta-regression of effect size for mean LDL-cholesterol on patient age, sex, and BMI did not yield significant results (online Supplementary eTable 6). There were insufficient studies to explore the influence of other moderators.

\section{HDL-cholesterol concentration}

HDL-cholesterol concentration was analysed using data from 15 studies comprising 1148 patients and 1387 controls. HDL-cholesterol was more variable in patients compared with controls $(\mathrm{CVR}=1.15 ; 95 \%$ CI $1.00-1.31 ; p<0.05)$ (Fig. 1 , online Supplementary eTable 2, eAppendix 11). Mean HDL-cholesterol was reduced in patients compared with controls $(\mathrm{SMD}=-0.26$; $95 \%$ CI -0.50 to $-0.01 ; p=0.04$ ) (Fig. 2, online Supplementary eTable 2, eAppendix 11). There was between-sample inconsistency $\left(I^{2}\right.$ value $=86.79 \%$; Cochrane $\left.Q=69.74, p<0.01\right)$. Funnel plot inspection (online Supplementary eAppendix 11) did not suggest publication bias (Egger $p=0.15$ ). Restricting analyses to antipsychotic naïve patients did not change CVR or SMD outcomes (online Supplementary eTable 3); however, restricting analyses to patients with schizophrenia led to a loss of statistical significance for the SMD $(p=0.17)$, but not the CVR finding (online Supplementary eTable 4). Exclusion of a single outlying study (online Supplementary eAppendix 11) for the SMD analysis did not alter results (online Supplementary eTable 5); no CVR outliers were identified. Meta-regression of effect size for mean HDL-cholesterol on patient age and BMI did not yield significant results (online Supplementary eTable 6). However, more males in study populations were associated with higher HDL-cholesterol effect size magnitudes ( $z=2.02, r=0.97, p=0.04)$ (Fig. 3). There were insufficient studies to explore the influence of other moderators.

\section{Triglyceride concentration}

Triglyceride concentration was analysed using data from 15 studies comprising 1072 patients and 1108 controls. There was no difference in triglyceride concentration variability between patients and controls $(\mathrm{CVR}=1.07 ; 95 \%$ CI $0.82-1.41 ; p=0.61$ ) (Fig. 2, online Supplementary eTable 2, eAppendix 12). However, mean triglyceride concentration was increased in patients compared with controls $(\mathrm{SMD}=0.23$; 95\% CI $0.10-0.36 ; p=0.0006$ ) (Fig. 2, online Supplementary eTable 2, eAppendix 12). There was between-sample inconsistency $\left(I^{2}\right.$ value $=45.89 \%$; Cochrane $Q=$ 26.63, $p=0.02$ ). Funnel plot inspection (online Supplementary eAppendix 12) suggested publication bias was not significant (Egger $p=0.63$ ). Restricting analyses to antipsychotic naïve patients and those with schizophrenia did not change CVR or SMD outcomes (online Supplementary eTables 3 and 4). No outliers were identified. Meta-regression of mean triglyceride effect size on patient age and BMI did not yield significant results (online Supplementary eTable 6). However, fewer males in study population were associated with higher triglyceride effect size magnitudes $(z=-2.18, r=0.98, p=0.03$ ) (Fig. 3).

\section{Discussion}

Our first main finding is to systematically demonstrate greater variability in virtually all metabolic parameters examined in people with FEP compared with healthy controls, except for triglyceride levels. Antipsychotic treatment does not explain these findings as results remained significant when restricted to samples of antipsychotic naïve patients. Our second main finding is that greater symptom severity is associated with increased fasting glucose levels and female sex is associated with worse metabolic outcomes, namely increased insulin resistance, reduced HDL-cholesterol levels, and raised triglyceride levels. Our third main finding extends the outcomes of previous meta-analyses of mean differences in the field (Pillinger et al., 2017a, 2017b, 2019a) by confirming, with a larger data set, that patients with antipsychotic naïve FEP present with raised fasting glucose levels, glucose levels following the OGTT, fasting insulin levels, insulin resistance and triglyceride levels, and reduced levels of totalcholesterol. In contrast to previously less well-powered meta-analyses, we show that FEP is associated with reductions in HDL-cholesterol levels, and that there is no significant difference between patients and controls for LDL-cholesterol levels. These outcomes were robust to sensitivity analyses that excluded outlying studies.

There is increasing interest in defining psychosis subtypes by peripheral biomarkers, and previous evidence of metabolic differences between patients based on symptom dimensions has been noted (Kirkpatrick, Fernandez-Egea, Garcia-Rizo, \& Bernardo, 2009). However, such studies have not addressed whether there is greater metabolic variability in people with psychotic disorders beyond what might be expected due to normal individual differences, with the result that any identified subtypes may be no different from subtypes present in the healthy population. Furthermore, studies that select patients by subtype may result 
in the sampling of extreme ends of a distribution of similar variance to that seen in controls. Our meta-analysis addresses these issues and extends our understanding of psychosis as a heterogenous disorder by showing, for the first time, altered metabolic variability in FEP.

\section{Interpretation}

One explanation for greater metabolic variability in patient groups may be due to greater variability in adherence to fasting conditions in clinical populations. However, this would not explain increased variability in $\mathrm{HbA}_{1 \mathrm{c}}$ levels seen in patients as this parameter is not affected by fasting; furthermore, in groups where fasting conditions were not uniformly maintained one would expect greater variability in triglyceride levels, which we did not observe. Alternatively, differences in variability could reflect heterogeneity in the biological processes underlying the disorder, indicating that metabolic dysregulation is seen only in some patients, or to varying degrees across patients. Our observation that female sex and more severe symptoms are associated with poorer metabolic outcomes indicates that sex differences and psychopathology may contribute to metabolic alterations in patients. To our knowledge, this is the first study to identify female sex as a potential risk factor for metabolic dysregulation in psychosis. Our observation of a relationship between increased total PANSS score and more severe glucose dysregulation has previously been observed in a large Chinese cohort of patients with antipsychotic naïve FEP (Lang, Li, \& Zhang, 2021). However, inspection of the scatter plot for meta-regression of symptom severity on glucose effect size suggests that the association may be driven by one study (online Supplementary eAppendix 4) and results should be interpreted in this context; this highlights the need for future studies to further investigate moderators of metabolic disturbance in psychosis.

\section{Implications}

The results of our meta-analysis build on recent studies exploring metabolic alterations in FEP; beyond emphasising that altered glucose and lipid homoeostasis is present from illness onset, we have identified that there is greater heterogeneity in metabolic parameter levels in patients than that in the healthy population. Meta-analysis of variability has shown that compared to placebo treatment, antipsychotic treatment is associated with greater variability in weight-gain in people with schizophrenia, which may reflect the presence of subgroups of patients who are more vulnerable to antipsychotic-induced metabolic disturbance (Neumeier et al., 2021). It is unclear if heterogeneity in antipsychotic-induced metabolic disturbance is related to heterogeneity in metabolic levels at onset of psychosis; identifying subgroups of patients from illness onset who are more likely to develop metabolic disease following antipsychotic prescription would allow for treatment stratification and precision medicine, and should be a key focus for future research (Pillinger et al., 2020).

Furthermore, understanding the determinants of metabolic variability could provide insight into the biological processes underlying psychosis. For example, a shared role for inflammation in insulin resistance and schizophrenia has been proposed (Perry et al., 2021); however, an inflammatory state may only be seen in a subgroup of patients (Pillinger et al., 2019b), which would explain the metabolic heterogeneity findings documented in the current study. Unfortunately, no studies included in the current meta-analysis provided inflammatory mediator data, precluding a meta-regression analysis exploring the moderating effects of such parameters on metabolic effect size magnitudes. Regardless of the underlying mechanism, recognising that female sex and more severe psychopathology is associated with poorer metabolic outcomes in FEP has implications for metabolic monitoring in clinical practice.

\section{Strengths and limitations}

Compared with previous meta-analyses assessing mean differences (Pillinger et al., 2017a, 2017b), this study has doubled the sample size for several parameters; this increased power provides greater confidence to the finding that antipsychotic naïve FEP is associated with metabolic dysregulation when assessed using mean differences in parameters.

By focusing on antipsychotic-naïve FEP, we limited duration of secondary illness-related factors that influence metabolic parameters. Furthermore, in a previous meta-analysis examining glucose dysregulation in FEP, sensitivity analysis examining studies in which participants were matched for diet and physical activity levels remained significant for raised fasting glucose levels in patients (Pillinger et al., 2017a). However, individuals in the prodromal state already have poorer dietary habits and decreased physical activity compared with age-matched controls, thus we cannot rule out residual confounding in the current meta-analysis (Koivukangas et al., 2010; Stubbs et al., 2016).

Diagnostic heterogeneity is recognised in FEP, which could contribute to variability outcomes. However, our sensitivity analysis of studies that examined only patients with schizophrenia did not change CVR outcomes (except for the total-cholesterol result), so we can be confident that variability results were not entirely the consequence of diagnostic heterogeneity across the psychosis spectrum. Inconsistency between studies was often moderate to high. This could reflect methodological factors, e.g. differences in assay sensitivity. However, the random effects model used is robust to inconsistency, and would not explain our variability findings, because these reflect within-study variation (where methodological factors are the same in both patient and control groups in any given study).

Although correction for multiple testing is not routinely performed in meta-analysis (Cumpston et al., 2019), we recognise that a series of meta-regression analyses were performed without correction; thus, these findings should be considered as hypothesis-generating and future studies are required to test these associations further. Furthermore, several meta-regression analyses were not possible owing to data not being presented, including the influence of ethnicity, illness duration, and proportion of smokers on mean difference and variability outcomes. Future studies should aim to examine these potential moderators.

\section{Conclusions}

Antipsychotic naïve patients with FEP present with both higher levels of glucose and insulin and greater variability in glucose, insulin, and lipid parameters relative to controls, suggesting that subgroups of patients may present with more severe metabolic changes than others. Furthermore, female sex and more severe psychopathology in FEP are associated with metabolic dysregulation. 
Supplementary material. The supplementary material for this article can be found at https://doi.org/10.1017/S0033291721005213

Financial support. Dr Pillinger is supported by the National Institute for Health Research (NIHR) and Maudsley Charity. Dr McCutcheon is supported by the NIHR. Professor Howes is supported by the Medical Research Council-UK (grant no. MC_A656_5QD30_2135), Maudsley Charity (grant no. 666), and Wellcome Trust (grant no. 094849/Z/10/Z), and the NIHR Biomedical Research Centre at South London and Maudsley NHS Foundation Trust and King's College London. The funders had no role in study design, data collection, data analysis, data interpretation, or writing of the report. The views expressed are those of the authors and not necessarily those of H Lundbeck A/s, the NHS/NIHR or the Department of Health.

Conflict of interest. Dr Pillinger has participated in educational speaker meetings organised by Lundbeck, Otsuka, Sunovion, Janssen, Schwabe Pharma, and Recordati. Dr McCutcheon has participated in educational speaker meetings organised by Otsuka and Janssen. Professor Howes is a parttime employee of $\mathrm{H}$ Lundbeck $\mathrm{A} / \mathrm{s}$ and has received investigator-initiated research funding from and/or participated in advisory/speaker meetings organised by Angellini, Autifony, Biogen, Boehringer-Ingelheim, Eli Lilly, Heptares, Global Medical Education, Invicro, Janssen, Lundbeck, Neurocrine, Otsuka, Sunovion, Rand, Recordati, Roche, and Viatris/Mylan. Professor Howes has a patent for the use of dopaminergic imaging. Professor Howes, Dr Pillinger, and Dr McCutcheon (and their families) do not have holdings or financial stakes in any pharmaceutical company.

\section{References}

Bowden, J., Tierney, J. F., Copas, A. J., \& Burdett, S. (2011). Quantifying, displaying and accounting for heterogeneity in the meta-analysis of RCTs using standard and generalised Q statistics. BMC Medical Research Methodology, 11, 41. doi: 10.1186/1471-2288-11-41

Brugger, S. P., \& Howes, O. D. (2017). Heterogeneity and homogeneity of regional brain structure in schizophrenia: A meta-analysis. JAMA Psychiatry, 74(11), 1104-1111. doi: 10.1001/jamapsychiatry.2017.2663

Crump, C., Winkleby, M. A., Sundquist, K., \& Sundquist, J. (2013). Comorbidities and mortality in persons with schizophrenia: A Swedish national cohort study. American Journal of Psychiatry, 170(3), 324-333. doi: 10.1176/appi.ajp.2012.12050599

Cumpston, M., Li, T., Page, M. J., Chandler, J., Welch, V. A., Higgins, J. P., \& Thomas, J. (2019). Updated guidance for trusted systematic reviews: A new edition of the Cochrane handbook for systematic reviews of interventions. Cochrane Database of Systematic Reviews, 10, ED000142. doi: 10.1002/ 14651858.ED000142

Egger, M., Zellweger-Zahner, T., Schneider, M., Junker, C., Lengeler, C., \& Antes, G. (1997). Language bias in randomised controlled trials published in English and German. Lancet (London, England), 350(9074), 326-329. doi: 10.1016/S0140-6736(97)02419-7

Greenhalgh, A. M., Gonzalez-Blanco, L., Garcia-Rizo, C., Fernandez-Egea, E., Miller, B., Arroyo, M. B., \& Kirkpatrick, B. (2017). Meta-analysis of glucose tolerance, insulin, and insulin resistance in antipsychotic-naive patients with nonaffective psychosis. Schizophrenia Research, 179, 57-63. doi: http://dx.doi.org/10.1016/j.schres.2016.09.026.

Higgins, J. P., Thompson, S. G., Deeks, J. J., \& Altman, D. G. (2003). Measuring inconsistency in meta-analyses. British Medical Journal, 327 (7414), 557-560. doi: 10.1136/bmj.327.7414.557

Kay, S. R., Fiszbein, A., \& Opler, L. A. (1987). The positive and negative syndrome scale (PANSS) for schizophrenia. Schizophrenia Bulletin, 13(2), 261276. doi: $10.1093 / \mathrm{schbul} / 13.2 .261$

Kirkpatrick, B., Fernandez-Egea, E., Garcia-Rizo, C., \& Bernardo, M. (2009). Differences in glucose tolerance between deficit and nondeficit schizophrenia. Schizophrenia Research, 107(2-3), 122-127. doi: 10.1016/j.schres.2008.09.023

Koivukangas, J., Tammelin, T., Kaakinen, M., Maki, P., Moilanen, I., Taanila, A., \& Veijola, J. (2010). Physical activity and fitness in adolescents at risk for psychosis within the northern Finland 1986 Birth Cohort. Schizophrenia Research, 116(2-3), 152-158. doi: 10.1016/j.schres.2009.10.022
Lang, X., Liu, Q., Fang, H., Zhou, Y., Forster, M. T., Li, Z., \& Zhang, X. (2021). The prevalence and clinical correlates of metabolic syndrome and cardiometabolic alterations in 430 drug-naive patients in their first episode of schizophrenia. Psychopharmacology (Berl), 238(12), 3643-3652. doi:10.1007/ s00213-021-05983-9.

Moher, D., Liberati, A., Tetzlaff, J., Altman, D. G., \& Group, P. (2009). Preferred reporting items for systematic reviews and meta-analyses: The PRISMA statement. Journal of Clinical Epidemiology, 62(10), 1006-1012. doi: 10.1016/j.jclinepi.2009.06.005

Neumeier, M. S., Homan, S., Vetter, S., Seifritz, E., Kane, J. M., Huhn, M., ... Homan, P. (2021). Examining side effect variability of antipsychotic treatment in schizophrenia spectrum disorders: A meta-analysis of variance. Schizophrenia Bulletin, 47(6), 1601-1610. doi: 10.1093/schbul/sbab078

Osby, U., Correia, N., Brandt, L., Ekbom, A., \& Sparen, P. (2000). Mortality and causes of death in schizophrenia in Stockholm County, Sweden. Schizophrenia Research, 45(1-2), 21-28. doi: 10.1016/s0920-9964(99)00191-7

Perry, B. I., Burgess, S., Jones, H. J., Zammit, S., Upthegrove, R., Mason, A. M., ... Khandaker, G. M. (2021). The potential shared role of inflammation in insulin resistance and schizophrenia: A bidirectional two-sample Mendelian randomization study. PLoS Medicine, 18(3), e1003455. doi: 10.1371/ journal.pmed.1003455

Perry, B. I., McIntosh, G., Weich, S., Singh, S., \& Rees, K. (2016). The association between first-episode psychosis and abnormal glycaemic control: Systematic review and meta-analysis. The Lancet. Psychiatry, 3(11), 10491058. doi: 10.1016/S2215-0366(16)30262-0

Pillinger, T., Beck, K., Gobjila, C., Donocik, J. G., Jauhar, S., \& Howes, O. D. (2017a). Impaired glucose homeostasis in first-episode schizophrenia: A systematic review and meta-analysis. JAMA Psychiatry, 74(3), 261-269. doi: http://dx.doi.org/10.1001/jamapsychiatry.2016.3803.

Pillinger, T., Beck, K., Stubbs, B., \& Howes, O. D. (2017b). Cholesterol and triglyceride levels in first-episode psychosis: Systematic review and meta-analysis. British Journal of Psychiatry, 211(6), 339-349.

Pillinger, T., D'Ambrosio, E., McCutcheon, R., \& Howes, O. D. (2019a). Is psychosis a multisystem disorder? A meta-review of central nervous system, immune, cardiometabolic, and endocrine alterations in first-episode psychosis and perspective on potential models. Molecular Psychiatry, 24(6), 776794. doi: 10.1038/s41380-018-0058-9

Pillinger, T., McCutcheon, R. A., Vano, L., Mizuno, Y., Arumuham, A., Hindley, G., ... Howes, O. D. (2020). Comparative effects of 18 antipsychotics on metabolic function in patients with schizophrenia, predictors of metabolic dysregulation, and association with psychopathology: A systematic review and network meta-analysis. The Lancet. Psychiatry, 7(1), 6477. doi: 10.1016/S2215-0366(19)30416-X

Pillinger, T., Osimo, E. F., Brugger, S., Mondelli, V., McCutcheon, R. A., \& Howes, O. D. (2019b). A meta-analysis of immune parameters, variability, and assessment of modal distribution in psychosis and test of the immune subgroup hypothesis. Schizophrenia Bulletin, 45(5), 1120-1133. doi: $10.1093 /$ schbul $/$ sby160

Pillinger, T., Osimo, E. F., de Marvao, A., Berry, M. A., Whitehurst, T., Statton, B., ... Howes, O. D. (2019c). Cardiac structure and function in patients with schizophrenia taking antipsychotic drugs: An MRI study. Translational Psychiatry, 9(1), 163. doi: 10.1038/s41398-019-0502-x

Reynolds, G. P. (2021). The etiology of metabolic disturbances in schizophrenia: Drugs, genes, and environment. International Journal of Neuropsychopharmacology, 24(10), 854-855. doi: 10.1093/ijnp/pyab047

Stroup, D. F., Berlin, J. A., Morton, S. C., Olkin, I., Williamson, G. D., Rennie, D., ... Thacker, S. B. (2000). Meta-analysis of observational studies in epidemiology: A proposal for reporting. Meta-analysis of observational studies in epidemiology (MOOSE) group. Journal of the American Medical Association, 283 (15), 2008-2012. doi: 10.1001/jama.283.15.2008

Stubbs, B., Firth, J., Berry, A., Schuch, F. B., Rosenbaum, S., Gaughran, F., ... Vancampfort, D. (2016). How much physical activity do people with schizophrenia engage in? A systematic review, comparative meta-analysis and meta-regression. Schizophrenia Research, 176(2-3), 431-440. doi: 10.1016/j.schres.2016.05.017

Viechtbauer, W., \& Cheung, M. W. (2010). Outlier and influence diagnostics for meta-analysis. Research Synthesis Methods, 1(2), 112-125. doi: 10.1002/ jrsm.11 